\title{
Enhancing Security for Mobile Ad hoc Networks by Using Identity Based Cryptography
}

\author{
Shohreh Honarbakhsh, Liza Binti Abdul Latif, Azizah bt Abdul Manaf, and Babak Emami
}

\begin{abstract}
This paper studies key management and security issues in mobile ad hoc networks (MANETs). Lack of strong identification results in unauthorized entities access to the sensitive data, and leads to loos of confidentiality and integrity, which causes many vulnerabilities and attacks in the network. We present the key management scheme as a combination of Identity-Based (User's Identification), Unique Transmission's time Factor and Threshold Cryptography for ad hoc networks. It is a certificateless solution which eliminates the need for public key distribution and certificates in public key management schemes. This scheme is also efficient in computation since small unique factor enhance the authentication of entities.
\end{abstract}

Index Terms-Identity based cryptography, key management, security, mobile ad hoc network.

\section{INTRODUCTION}

Mobile ad hoc network (MANET) provides communication for mobile devices which moved in any direction and manage arbitrarily. Nodes are self-configuring and communicate together directly or through intermediate nodes. Mobility characteristic of MANET results in dynamic topology which needs frequent changes in routing information. Nodes in ad hoc network acts as routers which causes network layer to be more vulnerable to security attacks Mobile Ad hoc Network is known as infrastructureless network [1].

Generally, routing protocols are classified into three categories: Proactive, Reactive and Hybrid protocol. In proactive protocol, all the nodes maintain routing table and routing information are always available. In reactive protocol, finding a route is done by route request rather than finding route in advance.

Mobility in MANET leads to lack of many security measures, which is used in conventional wireless network with the fixed infrastructure. Security attacks can be deployed in any network layers. Some physical tamper protection and transmission security procedures are capable for lowest layers while for higher layers protection cryptographic methods are applicable.

Security is defined as the reliable transmission of information through an insecure channel while the routing

Manuscript received November 14, 2013; revised January 22, 2014.

Shohreh Honarbakhsh and Azizah Bt Abdul Manaf are with the Advanced Informatics School, Universiti Teknologi Malaysia Kuala Lumpur, Malaysia (e-mail: shohreh_h2000@yahoo.com, Azizah07@citycamput.utm.my).

Liza Binti Abdul Latif is with the Center for Diploma Studies UTM SPACE, Universiti Teknologi Malaysia International Campuse (e-mail: liza@ic.utm.my).

Babak Emami is with the Universiti Teknologi Malaysia (e-mail: Eababak2@live.utm.my). protocol determines the route required to direct packets between the various devices in the mobile ad hoc network. In regards to some factors such as network environment, number of nodes, information transmission range of each node and so on, secured routing is difficult to provide.

Cryptography is a solution which provides most of the security requirements. Basically cryptographic methods are categorized into two groups [2]:

Symmetric Key Cryptography: In this method the key for encrypting and decrypting is the same. The algorithms of encryption and decryption are inversed of each other [2]. Symmetric key cryptography does not support the complete security requirements.

Asymmetric Key Cryptography: This method is also called public key cryptography. In Asymmetric Key Cryptography there are two types of keys; Public Key, and Private Key. Public Key is used for encrypting, and Private Key is used for decrypting. Sender encrypts the plaintext into ciphertext by its public key and the receiver decrypt the ciphertext into plaintext by its private key [2].

Based on the charactristics of mobile ad hoc network, functionality of Public Key infrastructure hase faced many challanges. Identity-based Cryptography is a form of asymmetric cryptography which is appropriate for MANET. In this method, third party server uses a simple and public identifier such as email address, for generating public key [3]. In identity-based cryptosystem, verification of user's validity is achieved by its unique identifier (ID). Private Key generates from a key generation center (KGC) while the Public Key is obtained from user's ID [4].

The Paper is organized as follows: Section II) briefly reviews the background of research on IBC methods, Section III) Desirable features of MANET Key Management Scheme, Section IV) Security Problems in MANET, Section V) Over view of Key Management scheme, Section VI) Proposed Algorithm, Section VII) Design IBC-t Method System Model, Section VIII) Experimental Result and finally in Section IX) concludes the research.

\section{RELATED WORK}

Most of the master key and private key generation schemes are derived from and are variants of Shamir's method [5].

Zhou et al. have suggested [6], a CA service of PKI can be distributed to multiple nodes in a MANET environment. This idea is also applicable to IBC.

In Identity-based cryptography, the PKG is a fundamental node which plays a crucial role for key generation.

Zhou et al. [6] proposed an idea that CA service of PKI is better to distributed to the multiple nodes in mobile ad hoc 
network.

Khalili et al. [7] for providing security in mobile ad hoc network proposed Identity-Based Cryptography. Khalili's scheme is based on Franklin and Bone scheme [5] and recognize the drawback of key management schemes with the assumption of existence of PKI and preexistence shared secret among nodes. In this scheme the Identity-Based method is combined with threshold cryptography scheme, which results in preparing a flexible key management method in mobile ad hoc network.

Deng et al. [6] proposed an Identity-Based key management and authentication for mobile ad hoc network employing IBC and threshold cryptography. This scheme includes distributed key generation and identity authentication. In this method assume that each node is able to discover its one-hop neighbor nodes and can get all the identity of nodes in the network. The key generation component provides the master public/private keys and public/private keys in a distributed way.

Zhang et al. [8] proposed a Distributed Private Key Generator (DPKG) to multiple nodes. In this scheme in offline manner, a master key is publishes to the DPKG. In this method, a threshold number of DPKGs act as PKG.

Li et al. [9] proposed a scheme based on signcryption that provides a secure transmission by applying key proxy, periodic private keys and multicast group of Private Key Generators. Key proxy derived from server nodes. Based on the location server nodes create multicast groups. Node send Route Request to server nodes group, when the node received a Route Reply select a server node with the shortest path as its proxy key. Afterward the routing information to the selected server node is preserved.

Feng et al. [10] proposed a method based on divide public key and private key into node specific and phase specific. In this scheme key update parameters is pre-distributed. Before network deployment, cryptographic parameters issue to each node. PKG publishes master keys to DPKG using threshold secret sharing. Each DPKG maintains a secret key and a set of values. Public key and private key is node specific and phase specific which the node specific is the first part of the key and the phase specific is the second part.

Ren et al. [11] proposed a scheme based on DPKG. In this method apply mutual authentication in public channel which leads to enhance the need of requirements of secure channel.

Lin et al. [12] proposed a hybrid method including traditional PKI and IBC. In this scheme key management integrate into secure routing protocols. This framework has two-layer hierarchical form. CA in the higher layer is responsible for external cluster domain authentication and the lower layer is responsible for internal cluster domain authentication by applying IBC.

Lee et al. [13] proposed a method based on using Key Generator Centre (KGC), which provide the privacy by multiple Key Privacy Authorities (KPAs). In this scheme KPS selects its own master key and compute its public key. The by collaboration among KPAs the system public key is calculated.

Deng et al. [14] introduce a method in which each node have an unique IP address or an Identity. This Identity obtain through auto configuration and dynamice address allocation, in condition that, this address does not have any confliction with other nodesn in the network. In this sceme eache node discover its one hop neighborhood in order to acquire the identities of other nodes in the network.

Nong Park[15] proposed an IBS method in which, sender node calculate security parameters and then sign this message digest before sending packet, receiver node compare security parameters and check the signature.

\section{The Advantages OF IBC To MAnet}

Easier to deploy without any infrastructure requirement. This saves certificate distribution, while bringing "free" pairwise keys without any interaction between nodes.

Its resource requirements, regarding process power, storage space, communication bandwidth, are much lower.

The public key of IBC is self-proving and can carry much useful information.

Secure routing is an important when transferring critical information between source and destination. Without a proper security method, a secured routing information and data transfer will be easily compromised.

\section{SECURITY PROBlems IN MANET}

One of the main challenges in MANET is to identify secure routing information. Mobile ad hoc networks eliminate the need for any infrastructure support by relying on the mobile wireless nodes themselves to collectively perform all networking functions, such as route discovery update or data transactions. The characteristics of ad hoc networks make them susceptible to numerous attacks. The wireless links are inherently vulnerable and compounded by irregular connectivity between the nodes due to the shared wireless channel.

Frequent connectivity problems are also caused by node mobility where nodes are free to leave the network, resulting in an unpredictable and dynamic network topology. These inherent vulnerabilities make it easier for attackers to compromise the networking infrastructure in the absence of robust security mechanisms. When an attacker succeeds to access information of another node illegally, the victim node is called the compromised node. Once node has been compromised, fake routing table can be distributed through the network and sensitive and critical information through these compromised nodes can be easily trapped.

Wireless devices are vulnerable to active and passive attacks. Secured routing information used to transfer data from one node to another node is difficult task to sustain [16].

Many solutions are proposed to solve these attacks including trust management and cryptography methods [17]. Cryptography mechanisms have played significant roles for providing security. Key management is one of the fundamental methods of cryptography that is set of techniques which support the generation, exchange, storage, use and replacement of keys (public/private key) among authorized entities. Identity-Based Cryptography (IBC), is a method that public key generated based on user's identity. Possibility of performing many attacks that comes from impersonation of legal users can be eliminated by this 
method.

\section{OVERVIEW OF Key MANAGEMENT}

Cryptographic methods are one of the significant ways in order to provide security in mobile ad hoc network. Key management in Identity-Based Cryptography includes generation, distribution, protection and revocation of the keys. In the following elaborated some methods of Identitybased Key Management in mobile ad hoc network [18].

\section{PRoposed AlgorithM}

The proposed algorithm for IBC-t method is defined as follows:

1) Start

2) Read UID/t

3) Read Data

4) Setup Phase $\rightarrow$ Master Public/Private Key

5) Extract Phase $\rightarrow$ Generate Public/Private Key

6) Encrypt Phase $\rightarrow$ Encrypt message

i. (Compute Master Public key and Public Key. $\left.M_{p k}, P u b_{k}, f\left(P u b_{k}\right), m \in M\right)$

ii. (Compute Master Private Key \& Private Key $\left.M_{s k}, \operatorname{Priv}_{k}, g\left(\operatorname{Priv}_{k}\right), c \in C\right)$

iii. $\quad\left(\operatorname{Priv}_{k} \rightarrow c\right)$

1. No: Discard Packet

2. Yes: Decrypt Packet

7) Decrypt Phase $\rightarrow$ Decrypt Message

8) End

\section{DESIGN IBC METHOD}

The proposed algorithm is described in this section. The main idea of this algorithm is applying unique and small parameter include identity $t_{i}$ and $u_{I D}$ in Identity-Based Cryptography method for increasing the performance of authenticity of entity. This attributes leads to reduce impersonating, packet dropping and routing attacks.

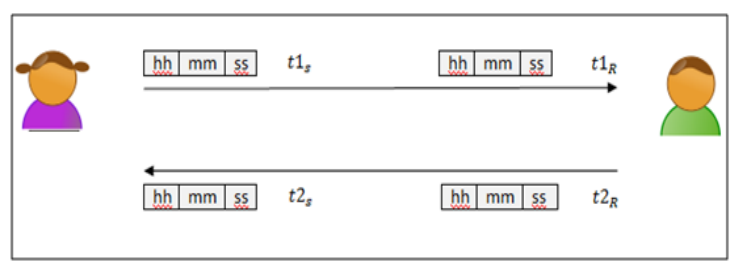

Fig. 1. Transaction for key management.

As Fig. 1 demonstrates, User A sends the request at the unique time $t 1_{s}$, and User $\mathrm{B}$ receives this request at the time $t 1_{R}$, this time might not be unique since many nodes in same distance can received this packet at the same time. On the other hand time $t 2_{R}$ is unique for User B.

In this scheme the public key is based on User ID and the unique time, this combination leads to reduce the possibility of impersonation and enhance the authentication process of user in the network.

In this scheme is:

$I D^{t i_{s} t i_{P K G}} \bmod p$, finding $t i_{S} t i_{P K G}$ is hard
Consider these assumption public/private key are as follow:

Master Public Key $=m_{P u b}$

Master Private Key- $\mathrm{K}(d, n)=m_{\text {Priv }}$

$d=1 /$ emod $(p-1)(q-1)$

Public key $=$ ID $\left\|t i_{s}\right\| m_{p u b}$

$T=t i_{s} t i_{P K G}$

Private Key= $\left(\text { ID } \| t i_{S}\right)^{T(1 / \mathrm{e} \bmod (p-1)(q-1))} \bmod n$

General process of the proposed model is depicted in Fig. 2.

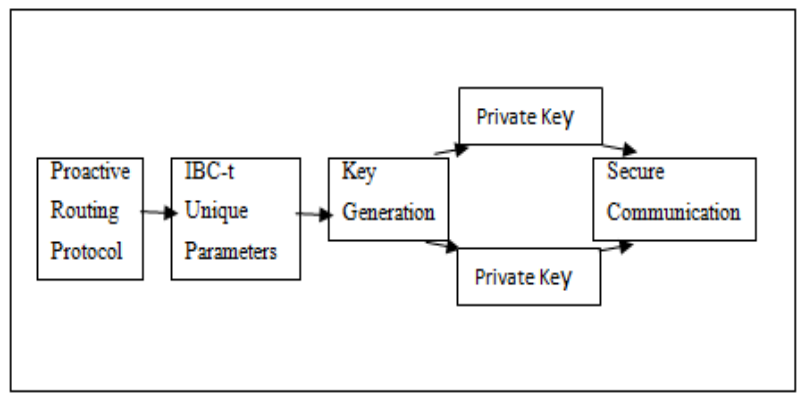

Fig. 2. Model process.

\section{SECURITY ANALYSIS}

In regard to the previous methods of IBC - their main ideas, parameters, and weaknesses - this research has more focused on eliminating the relevant vulnerabilities and enhancing the confidentiality and authenticity by proposing IBC-t.

A secure routing communication requires Confidentiality that preserves data secret to unauthorized entity, Integrity that impede data from alteration, Authentication that verifies data from authorized entity, and Non-repudiation that ensures an entity cannot deny their activities through communication.

One of the fundamental issues that this method point out is that during authentication process and communication there is not any trusted third party.

Secondly, in regard with Cluster heads are assumed as PKGs, we apply threshold for key management in the network. As a result single point of the failure is eliminated. It means if a mobile node within the network is compromised, the authentication still performed by other nodes. In addition a few packets are required to achieve the mutual authentication.

Moreover, in order to provide secure communication proper security countermeasures should be performed by applying strong authentication for message transmission. IBC-t proposed a method to authenticate message by using combination of ID and unique factor ' $t$ ' (transmission time).

There are many attacks in the network which comes from lack of strong authentication such as:

Impersonating another node to spoof route message; Advertising a false route metric to misrepresent the topology; Flooding Route Discover excessively as a DoS attack; Modifying a Route Reply message to inject a false route; Generating bogus Route Error to disrupt a working route; Suppressing Route Error to mislead others.

All these attack which mention in above caused unsecure routing in MANET. IBC-t proposed a strong user identification and authentication which leads to reduce the 
probability of these attacks by adding a small and unique factor which generates Public/Private keys in a reasonable time and does not lead to traffic or over head through the network.

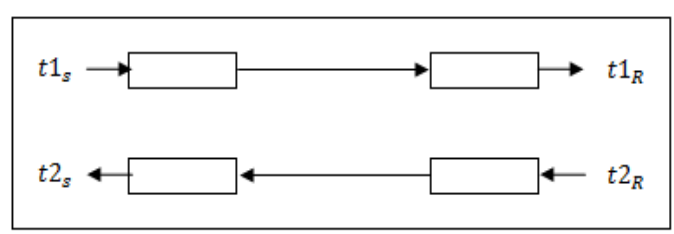

Fig. 3. Unique time in packet transaction.

Fig. 3 illustrated the transaction among two nodes, there is an unique time for sender and receiver $\left(t 1_{s}\right.$ for Sender and $t 2_{R}$ for Receiver). This unique small factor is combined with the User ID ( $t i_{\text {user }} \|$ User ID).

The combination of two unique user's identification leads to enhance the authentication of user and reduce the possibility of impersonation.

Another important factor in order to applying a suitable method to mobile ad hoc network is considering communication overhead.

In [15] communication overhead is considered by three methods ARAN, SRP and ISMANET.

ARAN introduce a preliminary certification process, through two phase, the first phase, a compulsory end to end authentication , and the second phase, an optional providing of shortest path. In this scheme each node sends a request of certification signed by a trusted certificate server before entering the network.

SRP proposed a method with the assumption that any of two nodes have a security association. In this method there is lack of authentication process for intermediate nodes between the source node and the destination node.

Nong Park proposed an IBS method in which, sender node calculates security parameters and then sign this message digest before sending packet, receiver node compare security parameters and check the signature.

The following Fig. 4 illustrates the communication overhead for these methods.

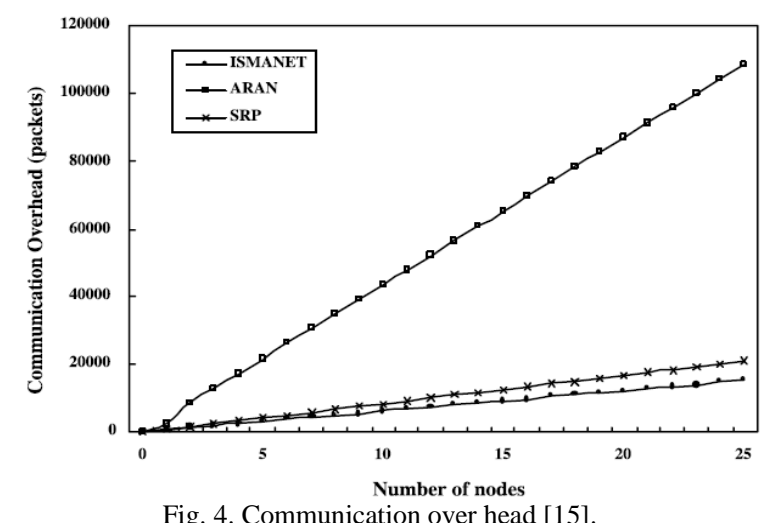

Compared to the results provided by [15], our proposed method does not cause a high communication over head through its process

Moreover, in regard with the applying a very small unique factor $t$ for improving identification, the latency is also covered by this proposed model.

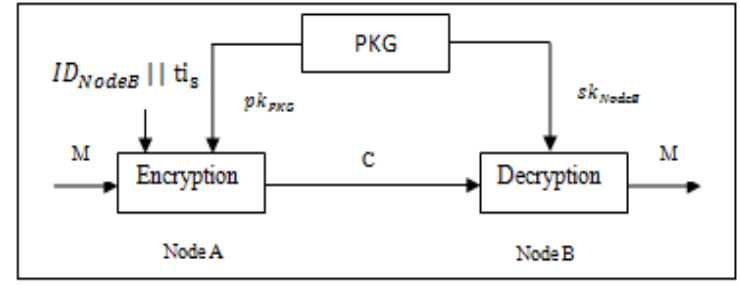

Fig. 5. Identity based scheme.

As Fig. 5 shows the combination between ID and $t_{i}$ does not incur a latency in the network.

Provide the strong authentication prepares the confidentiality and integrity in the network. Many active and passive attacks are happened because of lack of the strong authentication. Attacker interferes illegally through the communication and then can deny his activities easily if there is a weak identification and authentication process. Strong authentication will reduce impersonation attacks. Impersonation attack is a severe threat to the security of mobile ad hoc network [4]. If there is not such a proper authentication mechanism among the nodes, the adversary can capture some nodes in the network and make them similar non-malicious nodes. In this way, the compromised nodes can join the network as the benign nodes and begin to malicious activities, such as propagate fake routing information and gain inappropriate priority to access some confidential information.

\section{CONCLUSION}

One of the primary issues in mobile ad hoc network is applying key management. This research presents IBC-t method key management for MANET. IBC-t is a certificateless solution which allows public and private keys of mobile nodes proceed from combination of their known ID and some other factors while is simple and unique without complex computationally.

The concept of IBC-t method is a novel method of applying ID-Based public/private key which not only guarantees high-level authentication of mobile nodes but also facilitates efficient key generates which leads to resilience against node compromising.

Most existing security methods for mobile ad hoc networks are based on applying public key certificates. The finding of this research enhances authenticity and confidentiality through the network by reducing the computational time and enhancing the authentication of mobile nodes.

\section{REFERENCES}

[1] W. Mohammad and R. S. Kumar, "A survey of attacks happened at different layers of mobile Ad-Hoc network \& some available detection techniques," presented at the International Conference on Computer Communication and Networks, 2011.

[2] Z. Shushan and D. K. Robert, "An integrated key management and secure routing framework for mobile Ad-Hoc networks," presented at Tenth Annual International Conference on Privacy, Security and Trust, July 2012.

[3] S. Namita, "Secure communication using elliptic curve cryptosystem ad hoc network," University of Ottawa, 2008.

[4] C. Yu, Y. Mu, and S. Willy, "An identity-based broadcast encryption scheme for mobile ad hoc networks," Journal of Telecommunication and Information Technology, vol. 1, pp. 24-29, 2006. 
[5] D. Boneh and M. Franklin, "Identity-based encryption from the weil pairing," Lecture Notes in Computer Science, vol. 2139, pp. 213-229, 2001.

[6] L. Zhou and Z. J. Haas, "Securing ad hoc networks," IEEE Network, vol. 13, no. 6, pp. 24-30, 1999.

[7] A. Khalili, K. Jonathan, and A. A. William "Toward secure key distribution in truly ad-hoc networks," in Proc. SAINT Workshops, 2003, pp. 342-346.

[8] S. Xu and S. Capkun, "Distributed and secure bootstrapping of mobile ad hoc networks: framework and constructions," ACM Trans. Inf. Syst. Secur, vol. 12, no. 1, pp. 1-37, 2008.

[9] G. Li and W. Han, "A new scheme for key management in ad hoc networks," in Proc. 4th International Conference on Networking Proceedings, 2005, pp. 242-249.

[10] Y. Zhang, W. Liu, W. Lou, and Y. Fang, "Securing mobile ad hoc networks with certificateless public keys," IEEE Trans. Dependable Secur. Comput, vol. 3, no. 4, pp. 386-399, 2006.

[11] Y. Ren, J. Wang, Y. Zhang, and L. Fang, "Identity-based key issuing protocol for ad hoc networks," in Proc. International Conference on Computational Intelligence and Security, 2007, pp. 917-921.

[12] H. Y Lin, Y. M. Huang, and T. I. WANG, "Resilient clusterorganizing key management and secure routing protocol for mobile ad hoc networks," IEICE Transactions on Communications, vol. E88B, no. 9, pp. 3598-3613, 2005

[13] B. Lee, C. Boyd, E. Dawson, K. Kim, J. Yang, and S. Yoo, "Secure key issuing in ID-based cryptography," presented at Conferences in Research and Practice in Information Technology, 2004.

[14] D. Hongmei and M. Annindo, "Threshold and identity-based key management and authentication for wireless Ad Hoc networks," presented at International Conference on Information Technology, 2004.

[15] P. B. Nyong and L. Wonjun, "ISMANET: a secure routing protocol using identity-based signcryption scheme for mobile Ad-Hoc networks," IEICE Trans. Commun, 2005.

[16] C. Jin-Hee, "A Survey on trust management on mobile Ad hoc networks," Communications Surveys \& Tutorials, pp. 562-583, 2011.

[17] L. Wenjia and A. Joshi "Security issues in mobile Ad Hoc networks," 2006.

[18] S. Khan, "Passive security threats and consequences in IEEE 802.11 wireless mesh networks," International Journal of Digital Content Technology and its Applications, pp. 4-8, Dec 2008.

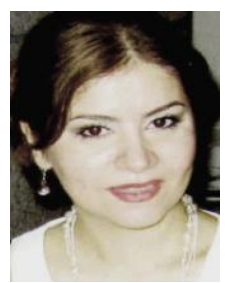

Shohreh Honarbakhsh was born in Iran, 1978. She is a Phd candidate in Advanced Information School, University Teknology Malaysia International Campus Kuala Lumpur. She obtained her bachelor's degree in computer science from Sadjad University of Mashad, and obtained a master's degree in computer science and information security at University Technology Malaysia (UTM), in 2011. Her research interests are wireless network security, cryptography, information security policy, cyber security, risk management and digital forensics.

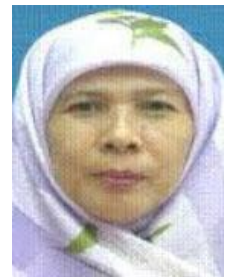

Liza Abdul Latiff was born in Singapore, 1961. She is an academician in Universiti Teknologi Malaysia for more than 30 years' experience. She obtained a diploma in electrical engineering (communication) in 1982 from Universiti Teknologi Malaysia and a bachelor of science in electrical engineering (honours) from South Dakota State University, USA in 1985. She came to work for a year and then went for study leave to pursue her masters. She graduated with masters in electrical engineering from Universiti Teknologi Malaysia in 1988. She came back to work teaching and doing research until 2003 when she went again for study leave pursuing her $\mathrm{PhD}$. She graduated in 2007 from Universiti Teknologi Malaysia proposing a new routing protocol in mobile ad hoc network that is energy efficient and better endto-end delay. She is currently the deputy dean at Centre of Diploma Studies managing and offering 16 programs in both engineering and nonengineering fields. The Centre currently has an enrolment of 1800 students and manages 130 academic and non-academic staff. Her research interests are wireless computer and data network focusing on routing protocols, security, quality of service and resource management algorithms.

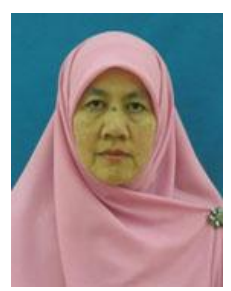

Azizah Abdul Manaf is a professor of image processing and pattern recognition from University Technology Malaysia (UTM). She graduated with B Eng. in Electrical 1980, MSc. computer science in 1985 and $\mathrm{PhD}$ in image processing in 1995. Her current areas of interest and research area are image processing, multimedia security and computer forensics. Currently she is supervising postgraduate students at the masters and $\mathrm{PhD}$ level in her research areas. She has also written numerous articles in journals and presented an extensive amount of papers at national and international conferences on her areas. Prof. Dr. Azizah has and is holding management positions at the University and Faculty level such as head of department, deputy dean, deputy director and academic director pertaining to academic development as well as on training for teaching and learning methodologies at the University.

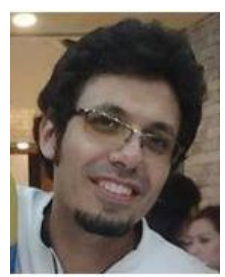

Babak Emami was born in Iran, 1983. He is a $\mathrm{PhD}$ candidate in University Teknology Malaysia International Campus Kuala Lumpur. He obtained a master's degree in computer science at University Technology Malaysia (UTM), in 2013. His research interest in wireless sensor network, mobile ad-hoc network, wireless body sensor network 